\title{
Pacific
}

Journal of

Mathematics

\section{PERMUTATION MODEL FOR SEMI-CIRCULAR SYSTEMS AND QUANTUM RANDOM WALKS}

PHILIPPE BIANE 


\title{
PERMUTATION MODEL FOR SEMI-CIRCULAR SYSTEMS AND QUANTUM RANDOM WALKS
}

\author{
Philippe Biane
}

\begin{abstract}
We give an approximation model for semi-circular families of non-commutative random variables, which is based on the permutation group. This model is compared to the random matrix model of Voiculescu, and a common refinement of the two approximations by explicit matrices with $0-1$ entries is exhibited.
\end{abstract}

\section{Introduction.}

The recent theory of semi-circular systems, introduced by D.Voiculescu [16] has proved to be a very efficient tool in the theory of free group factors (see e.g. [13] and the references therein), mainly due to the fact that semicircular systems can be approximated by gaussian random matrices [17]. It is thus an interesting problem to try to find other simple approximations of semi-circular systems. In this article we introduce such an approximation, which is based on permutation groups.

Let us describe briefly the key fact which is at the basis of this approximation. Let $\mathfrak{S}_{n+1}$ be the permutation group of $\{0,1, \ldots, n\}$, and for $1 \leq k \leq n$, let $\tau_{k} \in \mathfrak{S}_{n+1}$ be the transposition which exchanges 0 and $k$, and denote again by $\tau_{k}$ the corresponding left translation operator on $L^{2}\left(\mathfrak{S}_{n+1}\right)$, then the trace of the spectral measure of the operator $\frac{1}{\sqrt{n}}\left(\tau_{1}+\ldots+\tau_{n}\right)$ converges, as $n$ goes to infinity, towards the semi-circle distribution. This fact is a consequence of Theorem 1 below.

In the second part of the paper, we show how this permutation model is related to the gaussian random matrix model of Voiculescu. We first describe a convenient way of obtaining a gaussian hermitian matrix, which is to let a brownian motion evolve on the set of hermitian matrices. This brownian motion was first studied by Dyson, after Wigner's work on the semi-circle law. We show that the evolution of the spectrum of a brownian hermitian matrix is very much linked to the theory of tensor product representations: of the unitary groups. This enables us to approximate in law a hermitian random matrix by explicit finite dimensionnal matrices. This gives us a family of finite dimensionnal matrices depending on two integer parameters $n$ and $d$. The random matrix model of Voiculescu is obtained when we let 
$n$ go to infinity first and then $d$ go to infinity. The permutation model is obtained when $d$ goes first to infinity, and then $n$ does.

\section{A permutation model for semi-circular systems.}

1.1. Preliminaries. We first recall some definitions from Voiculescu [16].

1.1.1. Definition. A $\star$-non-commutative probability space $(A, \varphi)$ is composed of $A$ a unital $\star$-algebra and $\varphi$ a state on $A$.

Elements of $A$ are called (non-commutative) random variables.

In what follows, traces on matrix algebras will be normalized to be equal to 1 at the identity.

1.1.2. Let $I$ be an index set, and $\mathbb{C}\left(y_{\iota}, y_{\iota}^{*}\right)_{\iota \in I}$ be the free $\star$-algebra with generators $\left(y_{\iota}\right)_{\iota \in I}$. For every family $\left(X_{\iota}\right)_{\iota \in I}$ of elements of $A$ there is a unique

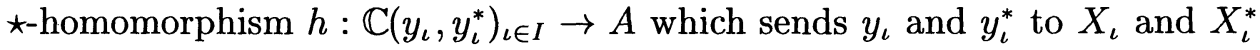
respectively.

Definition. The law of the family $\left(X_{\iota}\right)_{\iota \in I}$ is the map $\varphi \circ h$ on $\mathbb{C}\left(y_{\iota}, y_{\iota}^{*}\right)_{\iota \in I}$.

Convergence of a sequence of laws is defined as simple convergence on $\mathbb{C}\left(y_{\iota}, y_{\iota}^{*}\right)_{\iota \in I}$.

1.1.3. Definition. A family of $\star$-subalgebras $\left(A_{\iota}\right)_{\iota \in I}$ of $A$ is called free if, for all $n \geq 2, \varphi\left(a_{1} a_{2} \ldots a_{n}\right)=0$ whenever $a_{j} \in A_{\iota(j)}$ with $\iota(j) \neq \iota(j+1)$ for $1 \leq j \leq n-1$ and $\varphi\left(a_{j}\right)=0$ for $1 \leq j \leq n$. A family of random variables $\left(X_{\iota}\right)_{\iota \in I}$ is free if the family of $\star$-algebras $\left(A_{\iota}\right)_{\iota \in I}$ is free, where $A_{\iota}$ is the $\star$-algebra generated by $X_{\iota}$ for $\iota \in I$.

1.1.4. Definition. A family of random variables $\left(X_{\iota}\right)_{\iota \in I}$ is a semi-circular system if it is a free family, the $\left(X_{\iota}\right)_{\iota \in I}$ are self adjoint $\left(X_{\iota}=X_{\iota}^{*}\right)$ and $\varphi\left(X_{\iota}^{2 n}\right)=\frac{1}{n+1} \mathbf{C}_{2 n}^{n}, \varphi\left(X_{\iota}^{2 n+1}\right)=0$ for $n \in \mathbb{N}$.

\subsection{Permutations and semi-circular systems.}

1.2.1. Let $E$ be a countable set, and let $\mathfrak{S}(E)$ be the group of permutations of $E$ which fix all elements of $E$ except a finite number. The group $\mathfrak{S}(E)$ is the inductive limit of permutation groups of finite subsets of $E$.

Let $L(E)$ be the von Neumann algebra generated by the left regular representation of $\mathfrak{S}(E)$. It is a hyperfinite $I I_{1}$ factor, and its canonical trace is caracterized by $\operatorname{tr}(e)=1, \operatorname{tr}(\sigma)=0$ for $e \neq \sigma \in \mathfrak{S}(E)$ ( $e$ is the unit element of $\mathfrak{S}(E))$.

We consider elements of $\mathfrak{S}(E)$ as random variables in the non-commutative probability space $(L(E), t r)$.

1.2.2. Let $E=\{\delta\} \cup\left(\bigcup_{\iota \in I} E_{\iota}\right)$ be a partition of $E$ such that $\operatorname{card} E_{\iota}=\infty$ for all $\iota \in I$. We put $E^{\prime}=E \backslash\{\delta\}$. 
For $\iota \in I$, let $e_{\iota}^{1}, e_{\iota}^{2}, \ldots, e_{\iota}^{n}, \ldots$ be an enumeration of the elements of $E_{\iota}$, and let $\tau_{\iota}^{n}$, for $n \in \mathbb{N}$ be the element in $L(E)$ which corresponds to the transposition $\left(\delta, e_{\iota}^{n}\right)$.

Let $a_{\iota}^{n}=\frac{1}{\sqrt{n}} \sum_{k=1}^{n} \tau_{\iota}^{k} \in L(E)$, and let $\left(a_{\iota}\right)_{\iota \in I}$ be a semi-circular system in some non-commutative probability space $(A, \varphi)$.

\subsection{3.}

Theorem 1. The law of the family of random variables $\left(a_{\iota}^{n}\right)_{\iota \in I}$ in $(L(E), t r)$ converges towards the law of $\left(a_{\iota}\right)_{\iota \in I}$ as $n$ goes to infinity.

1.2.4. Before we proceed to the proof of the theorem in Section 1.3 we shall make some comments on this result.

For each $\varepsilon \in E^{\prime}$ let $G_{\varepsilon}$ be a copy of the group $\mathbb{Z} / 2 \mathbb{Z}$, let $\nu_{\varepsilon}$ be the non trivial element of $G_{\varepsilon}$ and let $G=\star_{\varepsilon \in E^{\prime}} G_{\varepsilon}$ be the free product group. There is a surjective morphism $G \rightarrow \mathfrak{S}(E)$ which sends $\nu_{\varepsilon}$ to the transposition $(\delta, \varepsilon)$. The elements $\nu_{\varepsilon}$ in the factor generated by the left regular representation of $G$ form a free family and if we replace in the definition of $a_{\imath}^{n}$ the transpositions $(\delta, \varepsilon)$ by $\nu_{\varepsilon}$ the Theorem 1.2.3 remains true, as follows from Voiculescu's central limit theorem $([\mathbf{1 5}])$.

More generally, let $H$ be a group with generators $\left(\theta_{\varepsilon}\right)_{\varepsilon \in E^{\prime}}$ satisfying $\theta_{\varepsilon}^{2}=e$ and such that there are surjective morphisms $\xi: G \rightarrow H$ and $\zeta: H \rightarrow \mathfrak{S}(E)$ with $\xi\left(\nu_{\varepsilon}\right)=\theta_{\varepsilon}$ and $\zeta\left(\theta_{\varepsilon}\right)=\tau_{\epsilon}$, then the theorem remains true if we replace $\tau_{\varepsilon}$ in the definition of $a_{\iota}^{n}$ by $\theta_{\varepsilon}$ considered as a left translation operator on $L^{2}(H)$. This means that, in some sense the relations between the transpositions $(\delta, \varepsilon)$ for $\varepsilon \in E^{\prime}$ "do not commute too much". This sentence may be clarified by the following remark. The transpositions $(0, k)$ for $1 \leq k \leq n$ form a system of generators of the permutation group of $\{0,1, \ldots, n\}$. Observe that, if we take as generators of this permutation group the transpositions $t_{k}=(k, k+1)$, for $0 \leq k \leq n-1$, then the limit as $n$ goes to infinity of the trace of the spectral measure of the operator $\frac{1}{\sqrt{n}}\left(t_{1}+\ldots+t_{n}\right)$ is the gaussian law.

\subsection{Proof of the Theorem 1.}

1.3.1. Let us first recall how to evaluate moments of semi-circular systems.

Definition. A partition $p=\left(V_{1}, \ldots, V_{l}\right)$ of the set $\{1, \ldots, m\}$ is called crossing if there exists $1 \leq k_{1} \neq k_{2} \leq l$ and $u_{1}, v_{1} \in V_{k_{1}}, u_{2}, v_{2} \in V_{k_{2}}$ such that $u_{1}<u_{2}<v_{1}<v_{2}$, and non-crossing in the opposite case.

This definition occurs in Kreweras [8], see also Speicher [14] where noncrossing partitions are called admissible.

For $m \in \mathbb{N}$, let $\mathcal{P}_{n c}(m)$ be the set of non-crossing partitions of $\{1, \ldots, m\}$ which consist of $\frac{m}{2}$ pairs, and for any $p \in \mathcal{P}_{n c}(m)$, choose a sequence $k_{1}, \ldots, k_{m}$ in $\{1, \ldots, m\}$ such that $p=\left(\left\{k_{1}, k_{2}\right\}, \ldots,\left\{k_{m-1}, k_{m}\right\}\right)$. 
Let $\left(a_{\iota}\right)_{\iota \in I}$ be a semi-circular system in $(A, \varphi)$, and $(\iota(1), \ldots, \iota(m))$ be a sequence of elements of $I$.

For $p \in \mathcal{P}_{n c}$, put

$$
V_{p}\left(a_{\iota(1)}, \ldots, a_{\iota(m)}\right)=\varphi\left(a_{\iota\left(k_{1}\right)} a_{\iota\left(k_{2}\right)}\right) \ldots \varphi\left(a_{\iota\left(k_{m-1}\right)} a_{\iota\left(k_{m}\right)}\right)
$$

(the expression for $V_{p}\left(a_{\iota(1)}, \ldots, a_{\iota(m)}\right)$ depends only on $p$ not on the choice of $\left.k_{1}, \ldots, k_{m}\right)$.

According to Speicher [14], for each sequence $\rho=(\iota(1), \ldots, \iota(m)) \in I^{m}$ one has $\varphi\left(a_{\iota(1)} \ldots a_{\iota(m)}\right)=0$ if $\mathrm{m}$ is odd and

$$
\varphi\left(a_{\iota(1)} \ldots a_{\iota(m)}\right)=\sum_{p \in \mathcal{P}_{n c}(m)} V_{p}\left(a_{\iota(1)}, \ldots, a_{\iota(m)}\right)
$$

if $m$ is even. Furthermore, for every $\iota, \kappa \in I$ one has $\varphi\left(a_{\iota} a_{\kappa}\right)=1$ if $\iota=\kappa$ and $\varphi\left(a_{\iota} a_{\kappa}\right)=0$ if $\iota \neq \kappa$.

This implies that

$$
\varphi\left(a_{\iota(1)} \ldots a_{\iota(m)}\right)=\operatorname{card}\left\{p \in \mathcal{P}_{n c}(m) \mid \iota\left(k_{1}\right)=\iota\left(k_{2}\right), \ldots, \iota\left(k_{m-1}\right)=\iota\left(k_{m}\right)\right\}
$$

for $m$ even.

1.3.2. We shall prove the Theorem 1 by showing that for each sequence $\iota(1), \ldots, \iota(m) \in I$ one has

$$
\lim _{n \rightarrow \infty} \operatorname{tr}\left(a_{\iota(1)}^{n} \ldots a_{\iota(m)}^{n}\right)=\varphi\left(a_{\iota(1)} \ldots a_{\iota(m)}\right)
$$

In order to evaluate the quantity $\operatorname{tr}\left(a_{\iota(1)}^{n} \ldots a_{\iota(m)}^{n}\right)$ we will first prove a sequence of lemma.

Let $\varepsilon_{1}, \ldots, \varepsilon_{m}$ be a sequence of elements of $E^{\prime}$, and $\tau_{\varepsilon_{k}}$ be the transposition $\left(\delta, \varepsilon_{k}\right)$ for $1 \leq k \leq m$.

The sequence $\left(\varepsilon_{k}\right)_{1 \leq k \leq m}$ determines a partition of $\{1, \ldots, m\}$ through the equivalence relation $k \sim l$ if and only if $\varepsilon_{k}=\varepsilon_{l}$.

Lemma 1. If the permutation $\tau_{\varepsilon_{1}} \tau_{\varepsilon_{2}} \ldots \tau_{\varepsilon_{m}}$ is the identity then the associated partition of $\{1, \ldots, m\}$ contains only sets of cardinal greater than 2 .

Proof. Suppose that for some $\eta \in E^{\prime}$, there exists exactly one $k$ such that $\varepsilon_{k}=\eta$, then the image of $\eta$ by the permutation $\tau_{\varepsilon_{1}} \tau_{\varepsilon_{2}} \ldots \tau_{\varepsilon_{m}}$ is not equal to $\eta$ (because $\tau_{\varepsilon_{1}} \ldots \tau_{\varepsilon_{k-1}}$ and $\tau_{\varepsilon_{k+1}} \ldots \tau_{\varepsilon_{m}}$ leave $\eta$ fixed), so that $\tau_{\varepsilon_{1}} \ldots \tau_{\varepsilon_{m}}$ cannot be the identical permutation.

Lemma 2. If the partition associated to $\varepsilon_{1}, \ldots, \varepsilon_{m}$ is composed of sets of cardinal 2 then $\tau_{\varepsilon_{1}} \ldots \tau_{\varepsilon_{m}}=e$ if and only if the partition is non-crossing.

Proof. First suppose that the partition of $\{1, \ldots, m\}$ is non-crossing. Let $k$ be such that $\varepsilon_{k}=\varepsilon_{k+1}$ (such a $k$ exists because of the properties of the 
partition), then $\tau_{\varepsilon_{1}} \tau_{\varepsilon_{2}} \ldots \tau_{\varepsilon_{m}}=\tau_{\varepsilon_{1}} \tau_{\varepsilon_{2}} \ldots \tau_{\varepsilon_{k-1}} \tau_{\varepsilon_{k+2}} \ldots \tau_{\varepsilon_{m}}$, and the partition associated to the sequence $\varepsilon_{1}, \ldots, \varepsilon_{k-1}, \varepsilon_{k+1}, \ldots, \varepsilon_{m}$ is again noncrossing and composed of sets of cardinal 2. From this observation, it is easy to prove by induction on even $m$ that $\tau_{\varepsilon_{1}} \tau_{\varepsilon_{2}} \ldots \tau_{\varepsilon_{m}}=e$ (since this is obviously true for $m=2$ ).

For the 'only if' part of the lemma, let $l$ be the greatest number such that $\varepsilon_{m-l}, \ldots, \varepsilon_{m}$ are all different elements of $E^{\prime}$ (one has $l \leq \frac{m}{2}$ because the partition is composed of pairs), and suppose $\varepsilon_{m-l-1}=\varepsilon_{r}$ with $r>m-l$, then the permutation $\tau_{\varepsilon_{m-l-1}} \tau_{\varepsilon_{m-l}} \ldots \tau_{\varepsilon_{m}}$ does not leave $\varepsilon_{r}$ fixed (because it sends $\varepsilon_{r}$ to $\varepsilon_{r-1}$ ). Since the permutation $\tau_{\varepsilon_{1}} \tau_{\varepsilon_{2}} \ldots \tau_{\varepsilon_{m-l-2}}$ does not contain any factor $\tau_{\varepsilon_{r}}$ it leaves $\varepsilon_{r}$ invariant, and so the product $\tau_{\varepsilon_{1}} \tau_{\varepsilon_{2}} \ldots \tau_{\varepsilon_{m}}$ cannot be the identity. We deduce from this that necessarily if $\tau_{\varepsilon_{1}} \tau_{\varepsilon_{2}} \ldots \tau_{\varepsilon_{m}}=e$ then $\varepsilon_{m-l-1}=\varepsilon_{m-l}$, so that $\tau_{\varepsilon_{1}} \tau_{\varepsilon_{2}} \ldots \tau_{\varepsilon_{m}}=$ $\tau_{\varepsilon_{1}} \tau_{\varepsilon_{2}} \ldots \tau_{\varepsilon_{m-l-2}} \tau_{\varepsilon_{m-l+1}} \ldots \tau_{\varepsilon_{m}}=e$. Now, if the partition associated to the sequence $\varepsilon_{1}, \ldots, \varepsilon_{m-l-2}, \varepsilon_{m-l+1}, \ldots, \varepsilon_{m}$ is non crossing, then so is the partition associated to $\varepsilon_{1}, \ldots, \varepsilon_{m-l-2}, \varepsilon_{m-l-1}, \varepsilon_{m-l}, \varepsilon_{m-l+1}, \ldots, \varepsilon_{m}$ because $\varepsilon_{m-l-1}=$ $\varepsilon_{m-l}$. The proof follows now by induction on even $m$.

1.3.3. We are now ready to finish the proof of the theorem. Let $\rho=$ $(\iota(1), \ldots, \iota(m))$ be a sequence in $I$. We compute the expectation $\operatorname{tr}\left(a_{\iota(1)}^{n} \ldots a_{\iota(m)}^{n}\right)$ by expanding the product. We find that

$$
\operatorname{tr}\left(a_{\iota(1)}^{n} \ldots a_{\iota(m)}^{n}\right)=n^{-\frac{m}{2}} \sum_{\varepsilon_{1} \ldots \varepsilon_{m}} \operatorname{tr}\left(\tau_{\varepsilon_{1}} \ldots \tau_{\varepsilon_{m}}\right)
$$

where the sum is taken over all sequences $\varepsilon_{1}, \ldots \varepsilon_{m}$ such that

$$
\varepsilon_{k} \in\left\{e_{\iota(k)}^{1}, \ldots, e_{\iota(k)}^{n}\right\} \text { for } 1 \leq k \leq m
$$

We call such a sequence a $\rho$-sequence.

Recall that the quantity $\operatorname{tr}\left(\tau_{\varepsilon_{1}} \ldots \tau_{\varepsilon_{m}}\right)$ is equal to 1 if $\tau_{\varepsilon_{1}} \ldots \tau_{\varepsilon_{m}}=e$ and 0 in the other cases.

To each sequence $\varepsilon_{1}, \ldots, \varepsilon_{m}$ in $E^{\prime}$ let us associate a partition of $\{1, \ldots, m\}$ as in 1.3.2. Let $p$ be a partition of $\{1, \ldots, m\}$ such that in each set there are at least 2 elements and in at least one of the sets there are strictly more than 2 elements, this partition is composed of at most $\frac{m-1}{2}$ sets, so that the number of $\rho$-sequences $\varepsilon_{1}, \ldots \varepsilon_{m}$ whose associated partition is $p$, is less than $n^{\frac{m-1}{2}}$. The contribution of such sequences to the expression (1) is therefore negligible as $n$ goes to infinity.

On the other hand, by Lemma 1 and 2, the $\rho$-sequences for which the associated partition contains at least one singleton, or which are crossing, 
give a contribution equal to 0 , so that it remains to count the contribution of the $\rho$-sequences whose partitions are non-crossing and composed of sets of cardinal 2.

Let $p=\left(\left\{k_{1}, k_{2}\right\}, \ldots,\left\{k_{m-1}, k_{m}\right\}\right)$ be a non-crossing partition of $\{1 \ldots, m\}$ composed of pairs. If there exists an $l$ such that $\iota\left(k_{2 l-1}\right) \neq \iota\left(k_{2 l}\right)$ then there is no $\rho$-sequence $\varepsilon_{1}, \ldots, \varepsilon_{m}$ such that $\varepsilon_{k} \in E_{\iota(k)}$ for $1 \leq k \leq m$ and whose associated partition is $p$. If $\iota\left(k_{2 l-1}\right)=\iota\left(k_{2 l}\right)$ for $1 \leq l \leq \frac{m}{2}$ then for $n \geq \frac{m}{2}$ the number of sequences $\varepsilon_{1}, \ldots \varepsilon_{m}$ with associated partition $p$, such that $\varepsilon_{k} \in\left\{e_{\iota(k)}^{1}, \ldots, e_{\iota(k)}^{n}\right\}$ for $1 \leq k \leq m$ is comprised between the two numbers $\left(n-\frac{m}{2}\right)^{\frac{m}{2}}$ and $n^{\frac{m}{2}}$.

From this we deduce that

$$
\operatorname{tr}\left(a_{\iota(1)}^{1} \ldots a_{\iota(m)}^{m}\right) \rightarrow \operatorname{card}\left\{p \in \mathcal{P}_{n c}(m) \mid \iota\left(k_{2 l-1}\right)=\iota\left(k_{2 l}\right) \text { for } 1 \leq l \leq \frac{m}{2}\right\}
$$

as $n$ goes to infinity. In view of 1.3 .1 , this proves the Theorem 1 .

\section{Relation with the gaussian matrix model.}

We will now show how the permutation model introduced in the preceding paragraph is related to the gaussian matrix model of Voiculescu. For this we will firstly describe a dynamic way of obtaining a random gaussian hermitian matrix, by letting a brownian motion evolve on the space of hermitian matrices.

\subsection{Brownian motion on hermitian matrices.}

2.1.1. The brownian motion on hermitian $d \times d$ matrices was studied by Dyson [5] after the work of Wigner on gaussian matrices and the semi-circle law ([18]). This is a stochastic process $X(t)=\left(X^{j k}(t)\right)_{1 \leq j, k \leq d}$ for $t \in \mathbb{R}_{+}$ such that for $1 \leq j<k \leq d,\left(X^{j k}(t)\right)_{t \in \mathbb{R}_{+}}$are independent complex brownian motions, $X^{j k}(t)=\bar{X}(t)^{k j}$, the processes $X^{j j}(t)$ are independent real brownian motions for $1 \leq j \leq d$, and the variances are given by $E\left[\left|X^{j k}(t)\right|^{2}\right]=t$ for $1 \leq j, k \leq d$.

Dyson investigated the stochastic process $\left(\lambda_{1}(t), \ldots, \lambda_{d}(t)\right)_{t \in \mathbb{R}_{+}}$whose components are the eigenvalues in increasing order of the matrix $X(t)$, and showed that it behaves like "a gas of brownian particles subject to repulsive Coulomb forces". This means, as shown by Mac-Kean [9], that this process satisfies a stochastic differential equation of the form

$$
d \lambda_{j}(t)=d B_{j}(t)+\sum_{k \neq j} \frac{d t}{\lambda_{j}(t)-\lambda_{k}(t)}
$$

the processes $B_{j}$ being independent brownian motions, or equivalently, that it is a diffusion process with generator $\frac{1}{2} \Delta+\operatorname{gradLog} h \nabla, \Delta$ being the usual 
Laplacian, $\nabla$ the gradient operator and $h$ the function $h(\lambda)=\prod_{1 \leq k<j \leq d}\left(\lambda_{J}-\right.$ $\lambda_{k}$ ) (cf [6]). If we observe that the function $h$ is positive, harmonic on the domain $\Omega=\left\{\lambda \in \mathbb{R}^{d} \mid \lambda_{j}>\lambda_{k}\right.$ for $\left.j>k\right\}$ and vanishes on its boundary, (see Lemma 3 below), then this generator can be put in the form $\frac{1}{2 h} \Delta(h$.) where $\Delta$ is the Laplacian on the domain $\Omega$ with Dirichlet boundary conditions. Probabilistically, this means that the process $(\lambda(t))_{t \in \mathbb{R}_{+}}$is obtained from brownian motion on $\mathbb{R}^{d}$ by a killing at the boundary of $\Omega$ followed by taking an $h$-process in the sense of Doob (cf [4]) with respect to the positive harmonic function $h$. Furthermore, one can show that this harmonic function is minimal, and corresponds to the unique Martin boundary point at infinity of the domain $\Omega$.

Lemma 3. The function $h$ is harmonic.

Proof. This proof comes from [1] Proposition 3. For every permutation $\sigma$ of $\{1 \ldots, d\}$ let $\sigma\left(\lambda_{1}, \ldots, \lambda_{d}\right)=\left(\lambda_{\sigma(1)}, \ldots, \lambda_{\sigma(d)}\right)$, then $h \circ \sigma=\operatorname{sgn}(\sigma) h$ and every polynomial which verifies this identity is a multiple of $h$. Since $\Delta$ commutes with every $\sigma$, one has again $\Delta h \circ \sigma=\operatorname{sgn}(\sigma) \Delta h$, and $\Delta h$ is a polynomial of lower degree than $h$, so that $\Delta h=0$.

As a corollary of the above remark, we can give an explicit expression of the semi-group of the process $\left(\lambda_{t}\right)_{t \in \mathbb{R}_{+}}$. Let $Q_{t}(x, y)$ be the density of this semi-group with respect to Lebesgue measure in the cone

$$
\left\{\lambda \in \mathbb{R}^{d} \mid \lambda_{j}>\lambda_{k} \text { for } j>k\right\},
$$

and $P_{t}(x, y)$ be that of the brownian semi-group in $\mathbb{R}^{d}$.

\section{Proposition 1.}

$$
Q_{t}(x, y)=\frac{h(y)}{h(x)} \sum_{\sigma \in W_{d}} \operatorname{sgn}(\sigma) P_{t}(x, \sigma(y)) .
$$

Proof. The semi-group of brownian motion killed at the boundary of the cone is given by the formula $\sum_{\sigma \in W_{d}} \operatorname{sgn}(\sigma) P_{t}(x, \sigma(y))$, (here $W_{d}$ is the Weyl group, that is the permutation group on $d$ letters acting on the coordinates of $\mathbb{R}^{d}$ ) as follows from the reflection principle, and the result is a consequence of Doob's definition of $h$-processes.

2.1.2. For our purposes, it will be more convenient to deal with brownian motion on traceless hermitian matrices. This is the orthogonal projection of the process $(X(t))_{t \in \mathbb{R}_{+}}$on the set of $d \times d$ matrices with zero trace. 
The process of eigenvalues of such a brownian motion is the orthogonal projection of the process $\left(\lambda_{t}\right)_{t \in \mathbb{R}_{+}}$on the set of elements of $\mathbb{R}^{d}$ which verify $\sum_{j} \lambda_{j}=0$. It is again obtained from brownian motion on the cone domain $\left\{\lambda \in \mathbb{R}^{d} \mid \lambda_{j}>\lambda_{k}\right.$ for $j>k$ and $\left.\sum_{j} \lambda_{j}=0\right\}$ by a killing at the boundary of the cone, followed by an $h$-transform with respect to the harmonic function $h$ restricted to this cone.

\subsection{Quantum random walks.}

In this section we shall describe a discrete non-commutative approximation of brownian motion on hermitian matrices, with the help of quantum random walks. More details about these processes can be found in $[2,10]$.

2.2.1. Let $G$ be a compact group, and $v N(G)$ be the von Neumann algebra generated by its left regular representation. For $g \in G$ call $\lambda_{g}$ the corresponding element of $v N(G)$.

Let $\phi$ be a continuous positive type function on $G$, with $\phi(e)=1$ where $e$ is the identity element in $G$. This function defines a state $\mu$ on $v N(G)$ caracterised by $\mu\left(\lambda_{g}\right)=\phi(g)$, and a completely positive map $T: v N(G) \rightarrow$ $v N(G)$ by the formula $Q\left(\lambda_{g}\right)=\phi(g) \lambda_{g}$.

Let $\mathcal{W}$ be the von Neumann algebra generated by the GNS representation of the infinite tensor product $v N(G)^{\otimes \infty}$ with respect to the infinite product state $\omega=\mu^{\otimes \infty}$, and $j_{n}: v N(G) \rightarrow \mathcal{W}$ be the morphisms defined by $j_{0}\left(\lambda_{g}\right)=$ $\operatorname{Id}_{\mathcal{W}}$ and $j_{n}\left(\lambda_{g}\right)=\lambda_{g}^{\otimes n} \otimes \operatorname{Id} \otimes \ldots \otimes I d \otimes \ldots$ for $n \geq 1$. The family of maps $\left(j_{n}\right)_{n \in \mathbb{N}}$ is called a quantum random walk. They form a dilation of the semi-group of completely positive maps $\left(T^{n}\right)_{n \in \mathbb{N}}$, which means that for each $n \in \mathbb{N}$ there is a conditionnal expectations $E_{n}$ from $\mathcal{W}$ onto the von Neumann subalgebra generated by the image of $j_{0}, j_{1}, \ldots, j_{n}$, and these conditionnal expectations satisfy the "Markov property" $E_{m} \circ j_{n}=j_{m} \circ T^{n-m}$ for $m \leq n$.

The semi-group $\left(T^{n}\right)_{n \in \mathrm{N}}$ is a non commutative analogue of a Markov semigroup. It is a convolution semi-group for the Hopf-von Neumann algebra structure of $v N(G)$ and the dilation $\left(j_{n}\right)_{n \in \mathbb{N}}$ is the analogue of a Markov process associated to this semi-group, which is a "non-commutative process with independent increments" (see Schürmann [12]).

2.2.2. Let $Z(v N(G))$ be the center of $v N(G)$. By Peter-Weyl theorem, this algebra is isomorphic to $L^{\infty}(\Gamma)$ where $\Gamma$ is the set of equivalence classes of irreducible representations of the group $G$. The restrictions of the maps $j_{n}$ to $Z(v N(G))$ take value in a commutative von Neumann subalgebra $\mathcal{X}$ - of $\mathcal{W}$.

If the function $\phi$ is central, then the maps $T^{n}$ send $Z(v N(G))$ into itself. Since they are completely positive maps, using the Peter-Weyl isomorphism $\chi: Z(v N(G)) \rightarrow L^{\infty}(\Gamma)$ we see that they give rise to a Markovian semi-group 
$\left(Q^{n}\right)_{n \in \mathbb{N}}$ on the set $\Gamma$, by the formula $Q^{n}=\chi \circ T^{n} \circ \chi^{-1}$. For $n \in \mathbb{N}$, let $\mathcal{X}_{n}$ be the von Neumann algebra generated by the images $j_{k}(Z(v N(G)))$ for $1 \leq k \leq n$, then the conditionnal expectation $E_{n}$ maps $\mathcal{X}$ onto $\mathcal{X}_{n}$. Since the algebra $\mathcal{X}$ is abelian there is a probability space $(\Omega, \mathcal{F}, P)$ with a filtration $\left(\mathcal{F}_{n}\right)_{n \in \mathrm{N}}$ and random variables $X_{n}$ with values in $\Gamma$, adapted to the filtration $\mathcal{F}_{n}$ which form a Markov process on $\Gamma$ with semi-group $\left(Q^{n}\right)_{n \in \mathrm{N}}$, there is an isomorphism $U: L^{\infty}(\Omega, \mathcal{F}, P) \rightarrow \mathcal{X}$ such that $U\left(L^{\infty}\left(\Omega, \mathcal{F}_{n}, P\right)\right)=\mathcal{X}_{n}$, the restriction of $E_{n}$ to $\mathcal{X}$ is equal to $U \circ E\left[. \mid \mathcal{F}_{n}\right] \circ U^{-1}\left(E\left[. \mid \mathcal{F}_{n}\right]\right.$ is the conditionnal expectation on the $\sigma$-field $\mathcal{F}_{n}$ ), the maps $j_{n}$ satisfy $U^{-1} \circ j_{n} \circ U=\mathfrak{X}_{n}$ where $\mathfrak{X}_{n}: L^{\infty}(\Gamma) \rightarrow L^{\infty}(\Omega, \mathcal{F}, P)$ is the morphism determined by $X_{n}$.

We can summarize this by saying that, in the case of a central function $\phi$, the construction in 2.2.1 of a quantum random walk gives a (commutative) Markov process on the set of classes of irreducible representations by restriction to the center.

2.2.3. Let $d$ be a positive integer, we specialize the preceding discussion to the case where $G=S U(d)$ and $\phi$ is the normalised character of the basic representation of $S U(d)$ (i.e. $\phi(g)=\operatorname{tr}(g)$ where $g \in S U(d)$ is considered as a $d \times d$ matrix). By the Cartan-Weyl theory of representations of compact semi-simple Lie groups, the set $\Gamma$ of classes of irreducible representations of $G$ can be identified with the set of dominant weights of the Lie algebra $s u(d)$. A dominant weight of $s u(d)$ can be identified with an integer vector $\lambda=\left(\lambda_{1}, \ldots, \lambda_{d}\right)$ such that $\lambda_{1} \leq \ldots \leq \lambda_{d}$ modulo an integer multiple of the vector $\xi=(1, \ldots, 1)$, or equivalently with its projection on the orthogonal of $\xi$ in $\mathbb{R}^{d}$.

The Markov chain on $\Gamma$ which has been associated to $\phi$ in 2.2 .2 can now be described in the following way (see [2]). First consider the random walk on $\mathbb{Z}^{d}$ whose jumps have a law which is uniform on the vectors $e_{i}-e_{i+1}, i=1, \ldots d-$ 1 and $e_{d}-e_{1}$ (where $\left(e_{1}, \ldots, e_{d}\right)$ is the canonical basis of $\left.\mathbb{Z}^{d}\right)$. Since the jumps are orthogonal to the vector $\xi$ this random walk gives rise to a random walk on the orthogonal of $\xi$. Now kill this random walk at its first exit time of the Weyl chamber $\left\{\lambda \in \mathbb{Z}^{d} \mid \lambda_{1} \leq \ldots \leq \lambda_{d}\right\}$ modulo $\xi \mathbb{Z}$, and then take the $h$-transform associated with the harmonic function $h(\lambda)=\prod_{1 \leq j \leq k}\left(\lambda_{j}+j-\right.$ $\left.\lambda_{k}-k\right)$, the resulting process has the same transition kernel as the Markov chain obtained by restriction of the quantum random walk $\left(j_{n}\right)_{n \in \mathrm{N}}$ to the center of $v N(S U(d))$, when $\Gamma$ is identified with the set of dominant weights. (Remark: the integer $h(\lambda)$ is the dimension of the irreducible representation with highest weight $\lambda$ and it is a harmonic function of $\lambda$ for the transition operator of the random walk killed at the boundary of the cone.)

It is not difficult to prove, using standard weak convergence arguments 
(see Pitman [11] for the case of $S U(2)$ ), that the process $\left(\frac{\lambda_{[n t]}}{\sqrt{n}}\right)_{t \in \mathbb{R}_{+}}$(where $[x]$ denotes the integer part of $x$ ) converges in law (on the usual Skorokhod space cf Jacod, Shyriaev [7]) towards the law of the motion of eigenvalues of the brownian motion on traceless hermitian matrices, which was described in Section 2.1.2. We shall state below (Sect. 2.2.4) a result which extends this convergence to include the whole quantum random walk, at the cost of obtaining only convergence of moments.

2.2.4. The finite dimensionnal representations of $S U(d)$ and $s l(d, \mathbb{C})$ are in one to one correspondance, so we will use the Lie algebra $s l(d, \mathbb{C})$ instead of the compact group $S U(d)$ in what follows.

Let $\left(E_{j k}\right)_{1 \leq j, k \leq d}$ be a family of matrix units in $M_{d}(\mathbb{C})$ and let $H^{j k}=$ $E_{j k}$, for $1 \leq j \neq k \leq d$ and $H^{j j}=E_{j j}-\frac{1}{d}$ Id for $1 \leq j \leq d$, then $\left(H^{j k}\right)_{1 \leq j, k \leq d,(j, k) \neq(d, d)}$ form a basis of the Lie algebra $s l(d, \mathbb{C})$.

Let $\mathcal{U}(d)$ be the universal envelopping algebra of $s u(d)$. An element $P$ of the center $Z(\mathcal{U}(d))$ of $\mathcal{U}(d)$ acts by a constant $P(\lambda)$ on the space of an irreducible finite dimensionnal representation of $\operatorname{sl}(n, \mathbb{C})$ with highest weight $\lambda$, and $P(\lambda)$ is a symmetric polynomial function of $\lambda$ on $\left\{\lambda \in \mathbb{R}^{d} \mid \sum_{j=1}^{d} \lambda_{j}=0\right\}$. In this way we identify $Z(\mathcal{U}(d))$ with an algebra of symmetric polynomials.

If $P$ is a polynomial function, and $\alpha \in \mathbb{R}$, let $P^{\alpha}$ be the polynomial function obtained by scaling the variables by a factor $\alpha$.

Let $\gamma$ be a non zero finite dimensionnal representation of $s l(d, \mathbb{C})$, of dimension $r$, and $\gamma^{\otimes n}$ the Lie algebra tensor product representation

$$
\left(\gamma^{\otimes n}(u)=\sum_{k=1}^{n} \mathrm{Id}^{\otimes(k-1)} \otimes \gamma(u) \otimes \mathrm{Id}^{\otimes(n-k)}\right) .
$$

The representations $\gamma^{\otimes n}$ are extended as representations of the algebra $\mathcal{U}(d)$.

Let $v=\operatorname{tr}\left(\gamma\left(H^{j k}\right) \gamma\left(H^{k j}\right)\right)$ for $1 \leq j<k \leq d$, then the value of $v$ does not depend on $(k, j)$ and $v=\frac{d}{d-1} \operatorname{tr}\left(\gamma\left(H^{j j}\right) \gamma\left(H^{j j}\right)\right)$ for $1 \leq j \leq d$ (here $t r$ is the normalized trace on $\left.M_{r}(\mathbb{C})\right)$.

Let $(\mathcal{Y}, \omega)$ be the infinite product von Neumann algebra $M_{r}(\mathbb{C})^{\otimes \infty}$ (with respect to the normalized trace on $M_{r}(\mathbb{C})$ ) with the infinite product state $\omega$, and define $s_{n}: \mathcal{U}(d) \rightarrow \mathcal{Y}$ by $s_{n}(u)=\gamma^{\otimes n}(u) \otimes \operatorname{Id} \otimes \ldots$

Theorem 2. The law of the family of random variables on $(\mathcal{Y}, \omega)$

$$
\left(\frac{1}{\sqrt{n v}} s_{[n t]}\left(H^{j k}\right), s_{[n t]}\left(P^{\frac{1}{\sqrt{n v}}}\right)\right)_{t \in \mathbb{R}_{+}, 1 \leq j, k \leq d, P \in Z(\mathcal{U}(d))}
$$

converges as $n$ goes to infinity towards the law of

$$
\left(\left(X^{j k}(t)\right), P(\lambda(t))\right)_{t \in \mathbb{R}_{+}, 1 \leq j, k \leq d, P \in Z(\mathcal{U}(d))},
$$


where $\left(X(t)=\left(X^{j k}(t)\right)_{1 \leq j, k \leq d}\right)_{t \in \mathbb{R}_{+}}$is a brownian motion on hermitian traceless matrices as defined in 2.1.2, and $\lambda(t)$ is its eigenvalue process.

2.2.5. We shall not need the full strength of this result in the sequel, so we will only prove a weaker statement, namely, the following Proposition 2 . The Theorem 2 can be proved by similar arguments.

Proposition 2. The law of the family of random variables on $(\mathcal{Y}, \omega)$, $\left(\frac{1}{\sqrt{n v}} s_{n}\left(H^{j k}\right)\right)_{1 \leq j, k \leq d}$ converges as $n$ goes to infinity towards the law of $\left(X(1)=\left(X(1)^{j k}\right)_{1 \leq j, k \leq d}\right)$ with the notations of Theorem 2 .

Thus, the $X^{j k}(1)$ for $j \neq k$ are complex, centered, independent gaussian variables such that $X^{j k}(1)=\bar{X}^{k j}(1)$, and $E\left[\left|X^{j k}(1)\right|^{2}\right]=1$, and the variables $X^{j j}$ (1) for $1 \leq j \leq d$ are real, centered, gaussian variables with covariance $E\left[X^{j j}(1) X^{k k}(1)\right]=-\frac{1}{d}$ if $j \neq k$ and $=1-\frac{1}{d}$ if $j=k$.

The proof of this result is essentially the same as the Proposition of 4) in [3] and relies on the following two simple lemma.

Lemma 4. Let $L \in \operatorname{sl}(2, \mathbb{C})$ be a self adjoint element, then the law of $\frac{1}{\sqrt{n}} s_{n}(L)$ converges towards the law of a centered gaussian random variable of variance $\operatorname{tr}\left(\gamma(L)^{2}\right)$.

Proof. One has

$s_{n}(L)=\gamma(L) \otimes \operatorname{Id} \otimes I d \ldots+I \otimes \gamma(L) \otimes I d \otimes \ldots+\ldots \mathrm{Id}^{\otimes n-1} \otimes \gamma(L) \otimes \operatorname{Id} \otimes \ldots$

The proof of the lemma is just an application of the usual central limit theorem to the commuting, self-adjoint centered random variables $\gamma(L) \otimes$ $\mathrm{Id} \otimes \mathrm{Id} \ldots, \mathrm{Id} \otimes \gamma(L) \otimes \mathrm{Id} \otimes \ldots, \ldots, \mathrm{Id}^{\otimes n-1} \otimes \gamma(L) \otimes \mathrm{Id} \otimes \ldots$

Using a polarization argument, we have the following corollary of Lemma 4.

Corollary. For any sequence $H^{j_{1} k_{1}}, \ldots, H^{j_{m} k_{m}}$ the symmetrized moments

$$
\omega\left(\frac{1}{n !} \sum_{\sigma} \prod_{q=1}^{m} \frac{1}{\sqrt{n}} s_{n}\left(H^{j_{\sigma(q)} k_{\sigma(q)}}\right)\right)
$$

(the sum is over permutations of $\{1, \ldots, m\}$ ) have a limit as $n$ goes to infinity which is $E\left[X^{j_{1} k_{1}}(1) \ldots X^{j_{m} k_{m}}(1)\right]$.

We now only have to prove the following lemma to finish the proof. 
Lemma 5. For any sequence $H^{j_{1} k_{1}}, \ldots, H^{j_{m} k_{m}}$, one has

$$
\lim _{n \rightarrow \infty} \omega\left(\prod_{q=1}^{m} \frac{1}{\sqrt{n}} s_{n}\left(H^{j_{\sigma(q)} k_{\sigma(q)}}\right)-\prod_{q=1}^{m} \frac{1}{\sqrt{n}} s_{n}\left(H^{j_{q} k_{q}}\right)\right)=0 .
$$

Proof. Let $\sigma$ be the transposition $(l, l+1)$. One has the following identity in $\mathcal{U}(d)$.

$$
\begin{aligned}
\prod_{q=1}^{m} H^{j_{\sigma(q)} k_{\sigma(q)}}-\prod_{q=1}^{m} H^{j_{q} k_{q}} & =\prod_{q=1}^{l-1} H^{j_{q} k_{q}}\left[H^{j_{l} k_{l}}, H^{j_{l+1} k_{l+1}}\right] \prod_{q=l+2}^{m} H^{j_{q} k_{q}} \\
& =\prod_{q=1}^{l-1} H^{j_{q} k_{q}}\left(\sum_{1 \leq u, v \leq d} c_{u, v}^{j_{l} k_{l}, j_{l+1} k_{l+1}} H^{u v}\right) \prod_{q=l+2}^{m} H^{j_{q} k_{q}}
\end{aligned}
$$

where $c_{u, v}^{j k, j^{\prime} k^{\prime}}$ for $1 \leq u, v, j, k, j^{\prime}, k^{\prime} \leq d$ are the structure constants of the Lie algebra $\operatorname{sl}(d, \mathbb{C})$. We thus find that

$$
\begin{aligned}
& \omega\left(\prod_{q=1}^{m} \frac{1}{\sqrt{n}} s_{n}\left(H^{j_{\sigma(q)} k_{\sigma(q)}}\right)-\prod_{q=1}^{m} \frac{1}{\sqrt{n}} s_{n}\left(H^{j_{q} k_{q}}\right)\right) \\
& =\frac{1}{\sqrt{n}}\left\{\sum_{1 \leq u, v \leq d} c_{u, v}^{j_{l} k_{l}, j_{l+1} k_{l+1}} \omega\left(\prod_{q=1}^{l-1} \frac{1}{\sqrt{n}} s_{n}\left(H^{j_{q} k_{q}}\right) \frac{1}{\sqrt{n}} s_{n}\left(H^{u v}\right) \prod_{q=l+2}^{m} \frac{1}{\sqrt{n}} s_{n}\left(H^{j_{q} k_{q}}\right)\right)\right\}
\end{aligned}
$$

now, using the corollary of Lemma 4 and an easy induction argument (compare [3]) we conclude that the expression between \{\} remains bounded as $n$ goes to infinity, and so have the proof of Lemma 5.

\subsection{A two parameter family of matrices.}

2.3.1. With the notations of 2.2 .4 , let us consider the $d r^{n} \times d r^{n}$ matrix

$$
M_{d, \gamma}^{n}=\sum_{1 \leq j, k \leq d} E_{j k} \otimes \frac{1}{\sqrt{n v d}} s_{n}\left(H^{j k}\right) .
$$

By Proposition 1, when $n$ goes to infinity, the law of this matrix, considered as a non-commutative random variable in $\left(M_{d r^{n}}(\mathbb{C}), t r\right)$ converges to that of a hermitian gaussian traceless matrix with variance $\frac{1}{d}$ (namely the law of $X\left(\frac{1}{d}\right)$ as in Theorem 2$)$.

Furthermore, if $\left(\gamma_{\iota}\right)_{\iota \in I}$ is a family of commuting representations of $s l(d, \mathbb{C})$ on $M_{r}(\mathbb{C})$, the law of the family of matrices $\left(M_{d, \gamma_{\iota}}^{n}\right)_{\iota \in I}$ converges towards the law of a family $\left(M_{d, \iota}\right)_{\iota \in I}$ of $d \times d$ hermitian gaussian traceless matrices with 
independent entries and variance $\frac{1}{d}$. When $d$ goes to infinity, by Voiculescu's theorem [17] (here the matrices are traceless, but this is a minor modification with respect to [17], which is negligible as $d$ goes to infinity), the law of the family of random variables $\left(M_{d, \iota}\right)_{\iota \in I}$ converges towards the law of a semicircular system.

2.3.2. Instead of making $n$ go to infinity first we will make $d$ go to infinity first. Let us now take $\gamma$ to be the contragredient representation, then $r=d$, and one gets the following matrices

$$
M_{d}^{n}=\frac{1}{\sqrt{n}}\left(\sum_{1 \leq j, k \leq d} \sum_{m=1}^{n} E_{j k} \otimes \mathrm{Id}^{\otimes m-1} \otimes E_{k j} \otimes \mathrm{Id}^{\otimes n-m}-\frac{1}{d} \mathrm{Id}\right) .
$$

These matrices operate on the Hilbert space $\left(\mathbb{C}^{d}\right)^{\otimes n+1}$. The permutation group of $\{0,1, \ldots, n\}$ operates on $\left(\mathbb{C}^{d}\right)^{\otimes n+1}$ by $\sigma\left(x_{0} \otimes \ldots \otimes x_{n}\right)=x_{\sigma(0)} \otimes$ $\ldots \otimes x_{\sigma(n)}$, and a simple computation shows that the matrix $\sum_{1 \leq j, k \leq d} E_{j k} \otimes$ $\mathrm{Id}^{\otimes m-1} \otimes E_{k j} \otimes \mathrm{Id}^{n-m}$ is just the matrix $\mathrm{t}_{m}^{d}$ of the transposition $(0, m)$ acting on $\left(\mathbb{C}^{d}\right)^{\otimes n+1}$. We conclude that $M_{d}^{n}$ is equal to $\frac{1}{\sqrt{n}}\left(\mathfrak{t}_{1}^{d}+\ldots+\mathfrak{t}_{n}^{d}-\frac{1}{d} \mathrm{Id}\right)$.

For $1 \leq m \leq n$ let $\tau_{m}$ be the image of the transposition $(0, m)$ in the von Neumann algebra generated by the left regular representation of the symmetric group of $\{0,1, \ldots, n\}$. This von Neumann algebra is a non commutative probability space, when equipped with the canonical trace.

Lemma 6. When $d$ goes to infinity the family of random variables $\left(\mathfrak{t}_{m}^{d}\right)_{1 \leq m \leq n}$ converges in law towards the law of the family $\left(\tau_{m}\right)_{1 \leq m \leq n}$.

Proof. It is enough to prove that for any non trivial permutation $\sigma$ the normalized trace of its action on the space $\left(\mathbb{C}^{d}\right)^{\otimes n+1}$ goes to zero as $d$ goes to infinity. But this trace is just $\frac{d^{c(\sigma)}}{d^{n+1}}$ where $c(\sigma)$ is the number of cycles of $\sigma$, as can be verified using the basis $\left(e_{i_{1}} \otimes \ldots \otimes e_{i_{n}}\right)_{1 \leq i_{1}, \ldots, i_{n} \leq d}$ (where $\left(e_{k}\right)_{1 \leq k \leq d}$ is the canonical basis of $\mathbb{C}^{d}$ ) to compute the trace, and so the lemma is proved, since $c(\sigma)<n+1$ for a non-identical permutation $\sigma$.

There is no difficulty in constructing a family $M_{d, \iota}^{n}$ for $\iota \in I$ if $I$ is finite, just take the Hilbert space $\mathbb{C}^{d} \otimes\left(\left(\mathbb{C}^{d}\right)^{\otimes n}\right)^{\otimes I}$ and construct a copy of the matrix $M_{d}^{n}$ on the component $\mathbb{C}^{d} \otimes\left(\mathbb{C}^{d}\right)^{\otimes n}$ corresponding to $\iota \in I$. The family of random variables $\left(M_{d, \iota}^{n}\right)_{\iota \in I}$ will converge in law as $d$ goes to infinity, towards the law of the family $\left(a_{\iota \in I}^{n}\right)$ with the notations of Theorem 1.

We can summarize the convergence schemes that we have just seen in the following diagram 


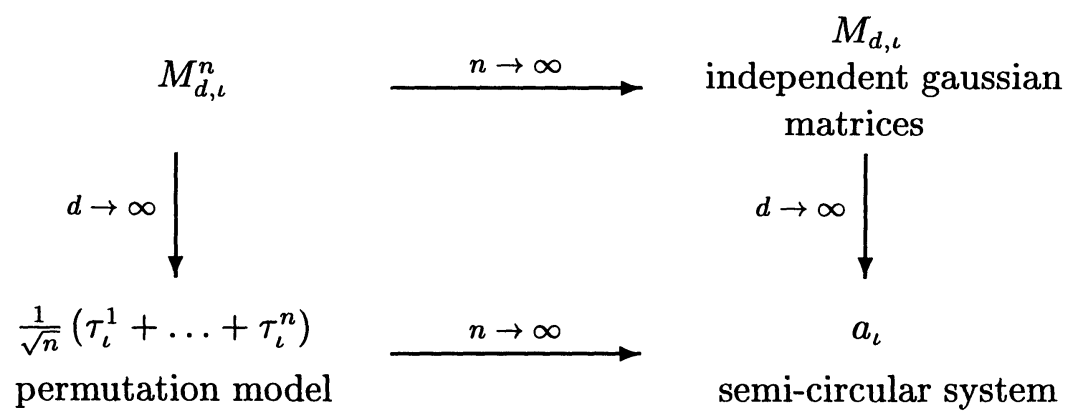

\section{References}

[1] P. Bérard and G. Besson, Spectres et groupes cristallographiques II, Ann. Inst. Fourier, 30 (3) (1980), 237-248.

[2] Ph. Biane, Quantum random walk on the dual of $S U(n)$, Prob. Th. and Rel. Fields, 89 (1991), 117-129.

[3] - Some properties of quantum Bernoulli random walks, Quantum probability and applications 6, L.Accardi, W. von Waldenfels ed., World scientific, 1991, 193204.

[4] J.L. Doob, Classical potential theory and its probabilistic counterpart, Springer Verlag, Berlin, Heidelberg, New York, 1984.

[5] F.J. Dyson, A brownian motion model for the eigenvalues of a random matrix, Journ. Math. Phys., 3 (1962), 1191-1198.

[6] N. Ikeda and S. Watanabe, Stochastic differential equations and diffusion processes, North holland, Kodansha, 1981.

[7] J. Jacod and A.N. Shyriaev, Limit theorems for stochastic processes, Springer, 1987.

[8] G. Kreweras, Sur les partitions non croisées d'un cycle Discrete Math., 1 (1972).

[9] H.P. Mac Kean, Stochastic integrals, Academic press, New York, 1969.

[10] K.R. Parthasarathy, A generalized Biane's process, Séminaire de probabilités XXIV, Springer Lecture Notes in Mathematics, 1426 (1990), 345-348.

[11] J.W. Pitman One dimensionnal brownian motion and the three dimensionnal Bessel process, Adv. Appl. Prob., 7 (1975), 511-526.

[12] M. Schürmann, Quantum stochastic processes with independent additive increments, Jour. Multivariate Anal., 38 (1991), 15-35.

[13] G. Skandalis, Algèbres de von Neumann de groupes libres et probabilités non commutatives [d'après Voiculescu et al.], Séminaire Bourbaki, exposé 764, Novembre 1992.

[14] R. Speicher, A new example of independence and white noise, Prob. Th. and Rel. Fields, 84 (1990), 141-159.

[15] D. Voiculescu, Symmetries of some reduced free product $C^{*}$-algebras, Operator algebras and their connection with topology and ergodic theory. Lect. Notes in Math. Springer, 1985, 556-588.

[16] - Circular and semi-circular systems and free product factors, Operator algebras, Unitary representations, envelopping algebras, and invariant theory, Progress 
in Mathematics, Volume 92, Birkhaüser, Boston, 1990.

[17] Limit laws for random matrices and free products, Invent. Math., 104 (1991), 201-220.

[18] E.P. Wigner, Characteristic vectors of bordered matrices with infinite dimensions, Ann. Math., 62 (1955), 548-564.

Received July 29, 1993.

C.N.R.S., Laboratoire de Probabilités Tour 56-66, $3^{e}$ ÉtAge

4, Place Jussieu, 75252, Paris Cedex 05

FRANCE 



\title{
PACIFIC JOURNAL OF MATHEMATICS
}

Founded by E. F. Beckenbach (1906-1982) and F. Wolf (1904-1989)

\section{EDITORS}

\author{
Sun-Yung Alice Chang (Managing Editor) \\ University of California \\ Los Angeles, CA 90095-1555 \\ pacific@math.ucla.edu
}

\section{F. Michael Christ}

University of California

Los Angeles, CA 90095-1555

christ@math.ucla.edu

Thomas Enright

University of California

San Diego, La Jolla, CA 92093

tenright@ucsd.edu

Nicholas Ercolani

University of Arizona

Tucson, AZ 85721

ercolani@math.arizona.edu
Robert Finn

Stanford University

Stanford, CA 94305

finn@gauss.stanford.edu

Vaughan F. R. Jones

University of California

Berkeley, CA 94720

vfr@math.berkeley.edu

Steven Kerckhoff

Stanford University

Stanford, CA 94305

spk@gauss.stanford.edu
Martin Scharlemann

University of California

Santa Barbara, CA 93106

mgscharl@math.ucsb.edu

Gang Tian

Courant Institute

New York University

New York, NY 10012-1100

tiang@taotao.cims.nyu.edu

V. S. Varadarajan

University of California

Los Angeles, CA 90095-1555

vsv@math.ucla.edu

\section{SUPPORTING INSTITUTIONS}

\section{CALIFORNIA INSTITUTE OF TECHNOLOGY \\ NEW MEXICO STATE UNIVERSITY \\ OREGON STATE UNIVERSITY \\ STANFORD UNIVERSITY \\ UNIVERSITY OF ARIZONA \\ UNIVERSITY OF BRITISH COLUMBIA \\ UNIVERSITY OF CALIFORNIA UNIVERSITY OF HAWAII}

\author{
UNIVERSITY OF MONTANA \\ UNIVERSITY OF NEVADA, RENO \\ UNIVERSITY OF OREGON \\ UNIVERSITY OF SOUTHERN CALIFORNIA \\ UNIVERSITY OF UTAH \\ UNIVERSITY OF WASHINGTON \\ WASHINGTON STATE UNIVERSITY
}

The supporting Institutions listed above contribute to the cost of publication of this Journal, but they are not owners or publishers and have no responsibility for its contents or policies.

Manuscripts must be prepared in accordance with the instructions provided on the inside back cover.

The Pacific Journal of Mathematics (ISSN 0030-8730) is published monthly except for July and August. Regular subscription rate: $\$ 215.00$ a year (10 issues). Special rate: $\$ 108.00$ a year to individual members of supporting institutions.

Subscriptions, orders for back issues published within the last three years, and changes of subscribers address should be sent to Pacific Journal of Mathematics, P.O. Box 4163, Berkeley, CA 94704-0163, U.S.A. Prior back issues are obtainable from Kraus Periodicals Co., Route 100, Millwood, NY 10546.

The Pacific Journal of Mathematics at the University of California, c/o Department of Mathematics, 981 Evans Hall, Berkeley, CA 94720 (ISSN 0030-8730) is published monthly except for July and August. Second-class postage paid at Berkeley, CA 94704, and additional mailing offices. POSTMASTER: send address changes to Pacific Journal of Mathematics, P.O. Box 6143, Berkeley, CA 94704-0163.

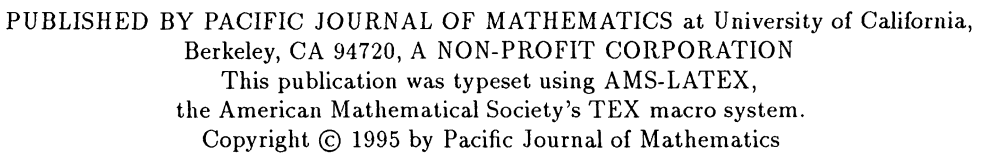




\section{PACIFIC JOURNAL OF MATHEMATICS}

\section{Volume $171 \quad$ No. $2 \quad$ December 1995}

On $H^{p}$-solutions of the Bezout equation

ERIC AMAR, JOAQUIM BRUNA FLORIS and ARTUR NiCOLAU

Amenable correspondences and approximation properties for von Neumann algebras

309

Claire ANANTHARAman-DElaroche

On moduli of instanton bundles on $\mathbb{P}^{2 n+1}$

VinCENZO ANCONA and Giorgio MaRia OtTAVIANI

Minimal surfaces with catenoid ends

JORGEN BERGLUND and WAYNE ROSSMAN

Permutation model for semi-circular systems and quantum random walks

PHILIPPE BIANE

The Neumann problem on Lipschitz domains in Hardy spaces of order less than one

RUSSELL M. BROWN

Matching theorems for twisted orbital integrals

409

REBECCA A. HERB

Uniform algebras generated by holomorphic and pluriharmonic functions on strictly

pseudoconvex domains

ALEXANDER IzZO

Quantum Weyl algebras and deformations of $U(g)$

NAIHUAN JING and JAMES ZHANG

Calcul du nombre de classes des corps de nombres

STÉPHANE LOUBOUTIN

On geometric properties of harmonic Lip ${ }_{1}$-capacity

PertTi MatTila and P. V. PARAmonov

Reproducing kernels and composition series for spaces of vector-valued holomorphic functions

Bent ØRsted and Genkai Zhang

Iterated loop modules and a filtration for vertex representation of toroidal Lie algebras

S. ESWARA RAO

The intrinsic mountain pass

MARTIN SCHECHTER

A Frobenius problem on the knot space

RON G. WANG

On complete metrics of nonnegative curvature on 2-plane bundles

DAVID YANG

Correction to: "Free Banach-Lie algebras, couniversal Banach-Lie groups, and more"

VLADIMIR G. PESTOV

Correction to: "Asymptotic radial symmetry for solutions of $\Delta u+e^{u}=0$ in a punctured disc"

KAI Seng (KAISing) CHOU (Tso) and Tom YAU-Heng WAN 